\title{
A pause for thought on lung cancer screening
}

$\mathrm{M}$ ore effective clinical strategies are badly needed to combat lung cancer, the leading cause of both cancer incidence and cancer death. A novel screening strategy based on low-dose computed tomography (CT) scanning is being studied in large randomized trials and may hold promise. However, the wide availability of CT threatens to undermine evidence-based practice if clinicians are inappropriately hasty in adopting CT screening. In our practices, we regularly encounter patients who inquire about or have already undergone CT-based screening. Some patient advocacy groups, such as the US Lung Cancer Alliance, have promoted this type of screening.

Advances in CT technology now permit imaging at sufficient resolution to identify lung nodules as small as a few millimetres in diameter while exposing patients to lower doses of radiation than would be required for a conventional thoracic CT scan. The largest and most prominent study to date of CTbased screening for lung cancer, the International Early Lung Cancer Action Program (I-ELCAP), ${ }^{2}$ has reported that screening with low-dose CT can successfully identify curable lung cancer at an early stage. The I-ELCAP protocol involved screening at baseline and annually thereafter. New nodules identified were investigated within a defined algorithm incorporating frequent $\mathrm{CT}$ scans, positron emission tomography scans, antibiotics or lung biopsy. Patients found to have cancer underwent surgical resection; the 10-year survival rate for these patients was $88 \%-92 \%$.

Given such favourable results, why shouldn't we rush to embrace CT-based screening for lung cancer? First, screening brings with it important unintended consequences. In the I-ELCAP study, screening was inefficient, identifying cancers in only $1.3 \%$ of participants. Other abnormalities, which were ultimately found to be benign but that required further workup, were 10-fold more common. Although the I-ELCAP protocol prevented unnecessary biopsies, patients were subjected to unnecessary anxiety while awaiting further testing.

However, an even more important consideration is that the apparent success demonstrated in CT screening studies for lung cancer may be an illusion. Screening for cancer is prone to several well-described types of bias, each of which could account for the study findings. The need to find cancers when they are small, although seemingly important, does not match the biology of all tumours. Rather than identifying cancers for which the potential for cure would otherwise have been missed, screening may merely provide earlier identification of cancers that remain curable after becoming clinically apparent (lead-time bias). Although screening increases the number of cancers diagnosed, many are slow-growing, indolent tumours (length-time bias). For some patients, screening may lead to overdiagnosis and overtreatment of cancers that would never become clinically evident because death from another cause is far more likely.
Clinicians ought to know better than to leap too early on the cancer screening bandwagon. We have been here before. Chest radiographs and sputum cytology were found to be ineffective screening tools for lung cancer after many years of research. ${ }^{3}$ Prostate-specific antigen testing is widespread in clinical practice, yet it has never been proven to reduce mortality from prostate cancer. ${ }^{4}$ Although a beneficial effect of mammography has been proven in older women, whether it reduces mortality from breast cancer in women aged 40-49 years is unknown. ${ }^{5}$ This highlights the consequences of failure to define optimal population subgroups for screening.

We acknowledge that proponents of CT screening may be right about its benefits. However, benefits need to be proven rather than assumed. The opportunity for proof will be lost if we allow premature enthusiasm to disseminate into widespread practice.

National and international professional organizations representing cancer, lung disease and medical imaging specialists could help by collaborating on joint policy statements to guide practice in the interim. Until then, if clinicians choose to offer CT screening for lung cancer, such decisions should be based on their best judgment of the incomplete evidence available and on the values and preferences of their patients, with forthright acknowledgement of the present uncertainty. Ideally, such patients should be enrolled in clinical trials of CT screening. Similarly, policy-makers should wait for better evidence of efficacy and cost-effectiveness before investing in more capacity for CT screening programs. In the meantime, given how much we know about the prevention of lung cancer, targeting smoking avoidance and cessation, rather than detection and management of lung cancer, would seem a better investment.

\section{Matthew B. Stanbrook MD PhD, Ken Flegel MDCM MSc CMAJ}

With the Editorial-Writing Team (Paul C. Hébert MD MHSc, Noni MacDonald MD MSc and Amir Attaran LLB DPhil)

Competing interests: See www.cmaj.ca/misc/edboard.shtml.

\section{REFERENCES}

1. Lung Cancer Alliance. Lung Cancer Alliance stands behind CT screening for lung cancer. Washington (DC): The Alliance; 2007. Available: www.lungcanceralliance .org/ACCPGuidelinesResponse9_12_07.htm (accessed 2009 Mar. 17).

2. International Early Lung Cancer Action Program Investigators; Henschke CI, Yankelevitz DF, Libby DM, et al. Survival of patients with stage I lung cancer detected on CT screening. N Engl J Med 2006;355:1763-71.

3. Manser RL, Irving LB, Stone C, et al. Screening for lung cancer. Cochrane Database Syst Rev 2004;(1):CD001991.

4. Lin K, Lipsitz R, Miller T, et al.; US Preventive Services Task Force. Benefits and harms of prostate-specific antigen screening for prostate cancer: an evidence update for the US Preventive Services Task Force. Ann Intern Med 2008;149:192-9.

5. Qaseem A, Snow V, Sherif K, et al.; for the Clinical Efficacy Assessment Subcommittee of the American College of Physicians. Screening mammography for women 40 to 49 years of age: a clinical practice guideline from the American College of Physicians. Ann Intern Med 2007;146:511-5 